\title{
Biomechanical evaluation of patellar tendon repair techniques: comparison of double krackow stitch with and without cerclage augmentation
}

\begin{abstract}
The purpose of this study was to evaluate two different repair techniques for the primary repair of patellar tendon ruptures. Using a cadaveric model, we biomechanically compared a double Krackow stitch with, and without cerclage augmentation. The specimens were tested at loads comparable to those likely to be encountered during an early rehabilitation program (approximately 40 Newtons) and for maximal mechanical parameters. The force at failure of the augmented repairs was $329 \pm 89$ Newtons $(\mathrm{N})$, while the non-augmented repairs failed at $278 \pm 87 \mathrm{~N}$. However, the force required to produce significant gapping at the repair site was not significantly different between both repair techniques. Additionally, there was no significant difference between the mean stiffness of the repairs in both groups within the loading range from 10-100N. However, as expected, at loads greater than 100 $\mathrm{N}$ the cerclage augmented repairs were significantly stiffer. Most notably, there was no gap formation in any of the constructs tested at forces $(40 \mathrm{~N})$ comparable to those seen during an early passive extension and active flexion program. These data suggest that it may be possible to achieve the benefits of an early rehabilitation program without augmentation of the repair, and thus avoid the potential complications arising from this additional material.
\end{abstract}

Volume 5 Issue I - 2016

\author{
Robin Gehrmann,' Robert Harten,' Regis \\ Renard,' Juluru P Rao,' Jacob A Spencer ${ }^{2}$ \\ 'University of Medicine and Dentistry of New Jersey, USA \\ ${ }^{2}$ Orthopaedic Surgery Resident Jersey City Medical Center \\ Jersey City, USA
}

Correspondence: Jacob A Spencer, Department of Orthopaedic Surgery, Jersey City Medical Center, 395 Grand Street, 3rd Floor, Jersey City, New Jersey 07302, USA, Tel 201915 2467, Fax 201-915-2025, Email jspencerdo@gmail.com

Received: May 04, 2016 | Published: May II, 2016

\section{Introduction}

Rupture of the extensor mechanism, the patellar tendon in particular, is an injury that occurs in two distinct patient populations as a result of a forceful quadriceps contraction against a flexed knee. This force has been estimated to be approximately 17.5 times body weight in healthy individuals. ${ }^{1}$ The first of these groups consists of young athletic individuals who rupture the ligament while involved in sporting activity. The second usually involves an older patient who may be taking corticosteroids, or has a systemic inflammatory disease process such as lupus, rheumatoid arthritis, or gout. Traditional repair techniques have centered on cerclage augmentation of the primary repair followed by a period of prolonged immobilization, typically six weeks. However, numerous studies have demonstrated the benefits of early mobilization and its positive effects on ligament and tendon healing as well as maintenance of articular cartilage. ${ }^{2-5}$

Treatment of this injury has evolved considerably over the last decade. As with many surgical procedures, there is a dilemma between immobilization required for healing and mobilization to preserve function. It is well known to orthopedists that prolonged immobilization increases the risk of scarring and muscle atrophy, weakens tendons, ligaments and bone leading to loss of strength and motion. ${ }^{3,6}$ We have also learned through histologic studies that extended immobilization has deleterious effects on articular cartilage. ${ }^{4,5}$ Motion promotes maintenance of normal cartilage composition while at the same time enhancing ligament and tendon healing. These injuries were originally treated with primary repair of the ligament, internal splinting to protect the repair, and cast immobilization. ${ }^{7-13}$ This technique also necessitated a second surgical procedure to remove the internal splint, and subjected the patient to a period of immobilization.

As the specialty of sports medicine evolves in concert with newer surgical techniques, a better understanding of rehabilitation and its effects on the healing process is similarly developing. This has led to accelerated rehabilitation programs with faster return to sports, or prior level of activity, and fewer complications related to prolonged immobilization. In an effort to decrease the need for prolonged immobilization and secondary surgical procedures newer suture techniques, as described by Krackow et al. ${ }^{14,15}$ and various other forms of non-removable augmentation have been described. These include the use of semitendinosus grafts. ${ }^{10,11,16,17}$ Dacron grafts, ${ }^{18,19}$ the Leeds-Keio ligament ${ }^{4}$ and no absorbable sutures including mersilene tape..$^{20-25}$ These varied techniques appear in the literature mostly as case reports, and are retrospective in design. The goal of these newer techniques is to eliminate the need for further operation. This has not always turned out to be the case however. Patients often complain of irritation from the suture material, specifically the cerclage stitch knot. Other complications related to tensioning of the cerclage material include loss of flexion and patellofemoral pain. These often require some form of surgical intervention. Increased surgical dissection required for augmentation, and larger amounts of foreign material can also theoretically increase the risk of a surgical complication. Therefore the goal of the present study was to determine if a construct can be achieved which is strong enough to withstand the rigors of early range of motion without the use of any augmentation material.

\section{Materials and methods}

\section{Specimen preparation}

Ten pairs of fresh frozen human cadaveric knees ( 5 male and 5 female) were stored at $-20{ }^{\circ} \mathrm{C}$, then thawed over 12 hours. The mean age of the donor knees was 69.8 years old, with a range of 39 to 84 . The extensor mechanism was then dissected leaving a portion of the quadriceps tendon, patella and patellar tendon intact with its tibial attachment. A simulated tendon rupture was produced by cutting through the substance of the tendon with a scalpel at the inferior pole of the patella. Both knees were repaired using a double Krackow stitch with number five Ethibond (Ethicon Inc., Somerville, NJ) through three patellar drill holes. One knee in each pair was augmented with a 
cerclage stitch placed posterior to the tibial tuberosity and through the quadriceps tendon using number five Mersilene tape.

\section{Biomechanical testing}

The specimens were mounted in a servo-hydraulic materialstesting system (MTS, Eden Prairie, Minnesota) by potting the tibial diaphysis in methyl methacrylate in a testing fixture, and using a custom made pneumatic grip to secure the quadriceps tendon. Tensioning the mounted specimen to $5 \mathrm{~N}$ and obtaining a reading from the extensometer determined the initial length of the repair. Each specimen was then pre-conditioned by cyclically loading to $40 \mathrm{~N}$ for 100 cycles, followed by loading to ultimate failure. All tests were conducted at a displacement rate of $25 \mathrm{~mm} / \mathrm{min}$. Deformation of the constructs was simultaneously monitored and recorded using an extensometer (MTS model 632.12C) with a gage length extender. An objective of this testing was to determine at what point there was significant gapping of the re-approximated tendon ends, and thus a likely clinical failure. Based on preliminary testing, it was found that a gap of approximately $3 \mathrm{~mm}$ consistently developed between the tendon ends as the total elongation of the construct reached $8 \mathrm{~mm}$, as determined by the extensometer. As this provided the least subjective and most consistent data, we elected to use an overall deformation of $8 \mathrm{~mm}$ to represent the clinical failure of the specimens.

The following five parameters were extracted from the mechanical testing data for comparison;

a) Total deformation of the repair at a force of $40 \mathrm{~N}$ after 100 cycles to $40 \mathrm{~N}$.

b) Force at clinical failure of the repair, defined as an $8 \mathrm{~mm}$ elongation of the repaired construct as compared to its initial length.

c) Stiffness of repaired construct in the range from 10 to $100 \mathrm{~N}$.

d) Stiffness of repaired construct from $100 \mathrm{~N}$ to ultimate failure.

e) Ultimate failure strength of the repair, defined as the maximum force sustained across the repaired construct.

\section{Statistical analysis}

The data from the mechanical tests were analyzed using paired Students T-tests for the five parameters listed above. Significance was determined at $\mathrm{P}$ values less than or equal to 0.05 .

\section{Results}

After completion of the cyclic testing, the non-augmented repairs demonstrated significantly greater levels of extension when tensioned to $40 \mathrm{~N}$ as compared to the Mersilene cerclage group $(6.3 \pm 2.8 \mathrm{~mm}$ Vs. $4.5 \pm 2.1 \mathrm{~mm}$, mean \pm standard deviation, $\mathrm{p}=0.03$ ), as seen in Figure 1. However, there was no visible gapping of the tendon ends in any of the constructs at any time during the cyclic testing. There was however, no significant difference in the mean force required for clinical failure of the repairs which was $178.6 \pm 96.7 \mathrm{~N}$ for the augmented group and $155.2 \pm 92.8 \mathrm{~N}$ for the non-augmented group (p $=0.27$ ), as shown in Figure 2. Similarly, although the mean stiffness of the augmented repairs between 10 and $100 \mathrm{~N}$ was slightly greater than the non-augmented group, this was not statistically significant $(37.0 \pm 14.3 \mathrm{~N} / \mathrm{mm}$ vs. $34.3 \pm 17.0 \mathrm{~N} / \mathrm{mm}, \mathrm{p}=0.38)$, as seen in Figure 3. However, beyond $100 \mathrm{~N}$ the mean stiffness of the augmented group $(38.9 \pm 10.0 \mathrm{~N} / \mathrm{mm})$ was significantly greater than the non-augmented group $(26.2 \pm 4.6 \mathrm{~N} / \mathrm{mm})$, (Figure 4$)$.
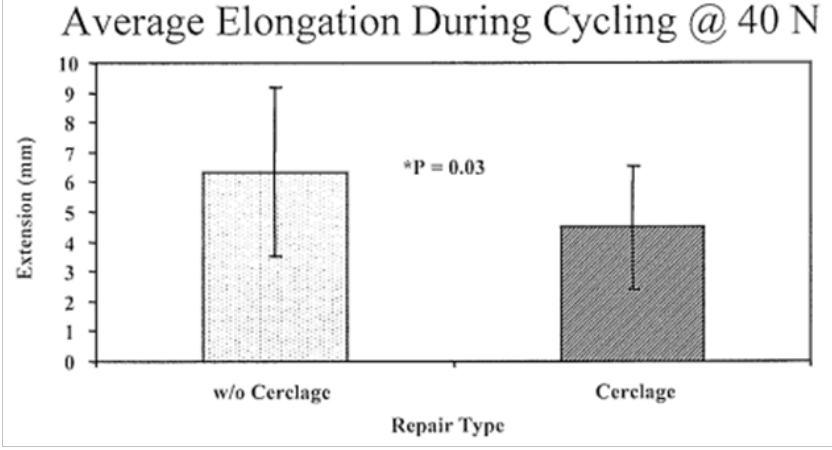

Figure I Average elongation of the patellar tendon constructs in the group augmented with cerclage and the non-augmented group (mean \pm standard deviation) after 100 cycles of loading to 40 Newtons. There was significantly greater elongation in the non-augmented group, $p=0.03$.

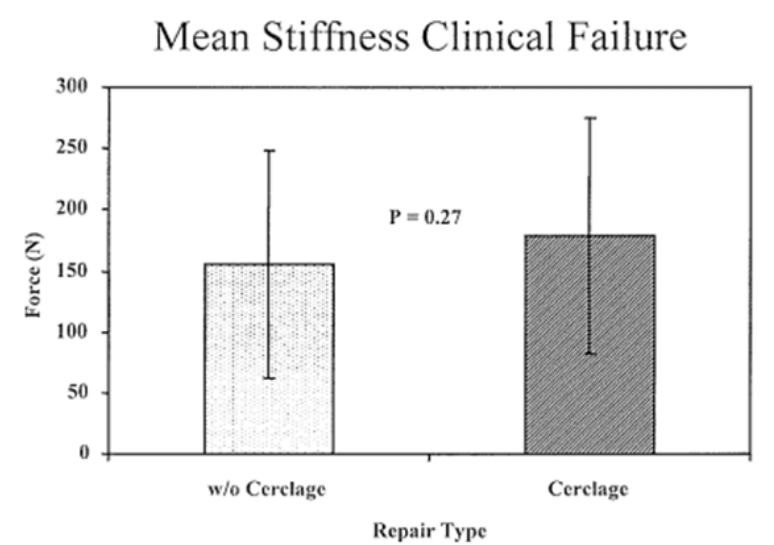

Figure 2 Average force (Newtons \pm standard deviation) required to produce $8 \mathrm{~mm}$ of elongation in the cerclage augmented repairs, and the non-cerclage group. There was no significant difference $(p=0.27)$ in the force required between these two groups.

$$
\text { Stiffness }(10-100 \mathrm{~N})
$$

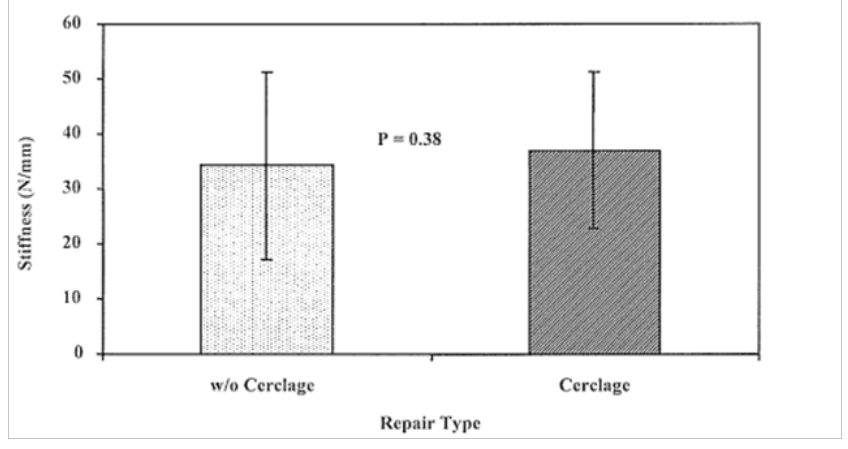

Figure 3 Mean stiffness ( $\mathrm{N} / \mathrm{mm} \pm$ standard deviation) of the repairs in both groups between 10 and 100 Newtons. There was no difference in stiffness between the two groups in this loading range, $p=0.38$.

As seen in Figure 5, there was no difference in the ultimate failure strength of the repairs between the two groups $(329 \pm 89 \mathrm{~N}$ vs. 278 $\pm 87 \mathrm{~N}, \mathrm{p}=0.09)$. There were no instances where a frank failure or rupture of the specimen occurred during the failure testing, which was stopped after $15 \mathrm{~mm}$ of elongation over the starting length. Instead, the repair materials (both the Ethibond and Mersilene) appeared to have been loaded beyond their yield point and simply "stretched out". 
There was also minimal cutting of the suture through the substance of the tendon and no failures associated with the cerclage materials or knot slippage. By the time the samples had reached their ultimate failure point, extensive gaping of the repair was typically observed.

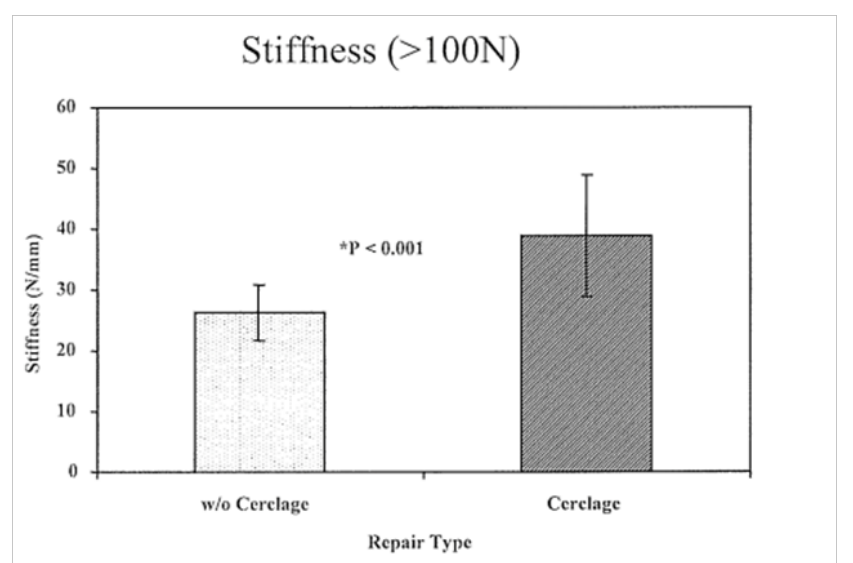

Figure 4 Mean stiffness $(\mathrm{N} / \mathrm{mm} \pm$ standard deviation) of the repairs in both groups between 100 Newtons and ultimate failure load. The cerclageaugmented repairs were significantly stiffer as compared to the nonaugmented group, $\mathrm{p}<0.00 \mathrm{I}$.

\section{Ultimate Failure}

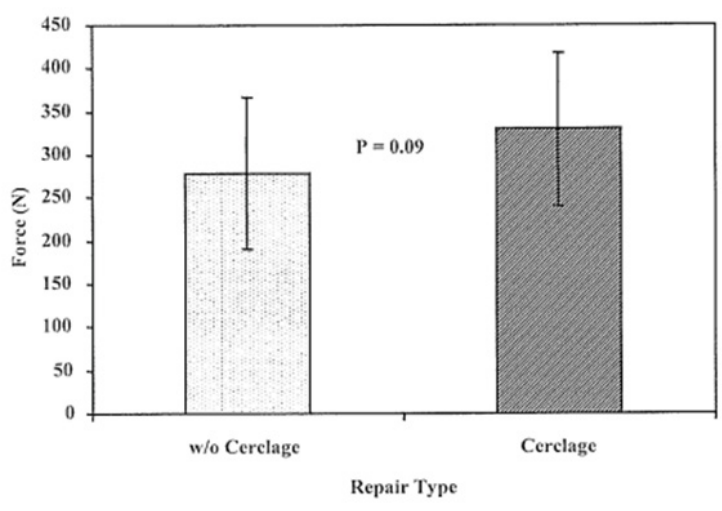

Figure 5 Ultimate failure load (mean \pm standard deviation) of the augmented and non-augmented repairs. The difference was not statistically significant, $P$ $=0.09$.

\section{Discussion and conclusion}

To date, Siwek and Rao ${ }^{11}$ published the largest series of extensor mechanism repairs roughly 20 years ago. ${ }^{11}$ Since that time, many newer techniques for the repair of extensor mechanism injuries have been presented as case reports and as conference proceedings. Several authors have advocated early motion with the use of a non-removable protective cerclage. These techniques are not however, without their own complications. The need for a second surgery to remove the cerclage or splint material secondary to patellofemoral problems and knot irritation has been reported by Lindy et al. ${ }^{21}$

The primary objective of this study was to compare a standardized repair technique using a double Krackow stitch both with and without cerclage augmentation at force levels estimated to exist across the tendon in an accelerated rehabilitation program consisting of active flexion with passive extension. We fully anticipated that at high force levels, the augmented repair would demonstrate superior biomechanical properties. However, to our knowledge there is no data in the literature that addresses the question of how strong a repair must be in order to begin early motion. For purposes of this study, we elected to set this critical value at $40 \mathrm{~N}$. This was judged to be conservative estimate as this value represents approximately one sixth of the force transmitted through the extensor mechanism during stair climbing, as estimated by the mathematical model of Heegaard et al. ${ }^{26}$

In this study, ultimate strength of the repaired constructs was defined as the maximum force the specimen withstood during testing. However, at this point the repair is clearly beyond what would result in a clinical failure, as there were typically gaps of over a centimeter between the tendon ends prior to reaching the peak force. As there is a paucity of literature dealing with the failure and healing of patellar tendon repairs, we based our "clinical failure" definition on the data available for the healing of other tendons. Gelberman et al.,,27 in dealing with the repair of flexor tendons, have established $3 \mathrm{~mm}$ as the maximum allowable gap distance for adequate tendon healing. Therefore, for the purposes of this study, we used $3 \mathrm{~mm}$ as a critical gap distance as well, although this is likely a very conservative estimate for patellar tendon healing.

Lindy et al. ${ }^{21}$ reported on 19 patients with a mean follow up of 22.4 months who underwent repair of acute disruptions of the knee extensor mechanism. The disruption was repaired with a single whipstitch using number 5 Ticron tied at the superior pole of the patella through two drill holes. This was reinforced with a 5-mm mersilene tape. Patients were allowed full weight bearing in a knee immobilizer or hinged knee brace with passive motion beginning on the first postoperative day. Two patients had prominent knots, one of which was removed surgically. Six patients developed patellofemoral chondrosis. Five of these had the Mersilene tape tied in full extension and all developed patellofemoral pain. All other patients had the Mersilene tied in ninety degrees of flexion and only one developed patellofemoral pain. In these patients, the Mersilene was providing no added protection until ninety degrees of flexion was reached. Marder et al. ${ }^{22}$ reported on 12 patients with acute traumatic patellar tendon ruptures repaired using a single Krackow whipstitch with no augmentation. All patients were started on active flexion to 45 degrees, and slide board assisted extensions by postoperative day number two. There were no reruptures in this series. There was also no loss of extension or extensor lag reported. Patellofemoral symptoms were present in five patients but activity was limited in only two.

The universal goal of any surgical technique is to facilitate a rapid return to function while minimizing potential complications. The results of the present study may provide important biomechanical data in support of the growing body of clinical evidence in favor of non-augmented patellar tendon repairs. It is important to point out that in no instance was there gapping of the tendon ends in either of the repair techniques at forces comparable to those seen during early passive extension and active flexion. There was also no significant difference in the forces at which significant gap formation occurred. The non-augmented repairs demonstrated significantly greater levels of extension after cyclic testing; however, there was no gap formation at the repair site. This may indicate that there is more "settling" of the non-augmented repairs after repeated cycling. However, after this takes place both repair groups behave similarly under low load conditions. This is further supported by the fact that the mean stiffness of the two repair techniques between $10-100 \mathrm{~N}$ was not significantly different. It may be necessary to compensate for this phenomenon during the repair process in order to avoid extensor lag, however, this is unclear, as problems of this type have not been reported in the previous studies. ${ }^{22,23}$ Although at loads greater than $100 \mathrm{~N}$ there was a significant difference in favor of augmented repairs, we would suggest 
that this difference is not of clinical significance. As these loads are greater than twice that which the repair will likely encounter in an early passive range of motion program, this finding may not be of any relevance.

In summary, with stronger suture materials now available and improved suturing techniques it may be possible to meet the biomechanical demands necessary to begin early range of motion without additional splinting of the repair. The data in this study suggest that it may be possible to achieve the benefits of early rehabilitation without augmentation of the repair.

\section{Acknowledgments}

None.

\section{Conflicts of interest}

None.

\section{References}

1. Zernicke RF, Garhammer J, Jobe FW. Human patellar-tendon rupture. $J$ Bone Joint Surg Am. 1977;59(2):179-183.

2. Gelberman RH, Botte MJ, Spiegelman JJ, et al. The excursion and deformation of repaired flexor tendons treated with protected early motion. J Hand Surg Am. 1986;11(1):106-110.

3. Noyes FR. Functional properties of knee ligaments and alterations induced by immobilization: a correlative biomechanical and histological study in primates. Clin Orthop Relat Res. 1977 ;(123):210-242.

4. Salter RB, Simmonds DF, Malcolm BW, et al. The biological effect of continuous passive motion on the healing of full-thickness defects in articular cartilage. An experimental investigation in the rabbit. $J$ Bone Joint Surg Am. 1980;62(8):1232-12351.

5. Salter RB, Bell RS, Keeley FW. The protective effect of continuous passive motion in living articular cartilage in acute septic arthritis: an experimental investigation in the rabbit. Clin Orthop Relat Res. $1981 ;(159): 223-247$.

6. Frank CB. Ligament Healing: Current Knowledge and Clinical Applications. J Am Acad Orthop Surg. 19964(1):74-83.

7. Donati RB, Cox S, Echo BS, et al. Bilateral simultaneous patellar tendon rupture in a female collegiate gymnast. A case report. Am J Sports Med. 1986;14(3):237-239.

8. Kuo RS, Sonnabend DH. Simultaneous rupture of the patellar tendons bilaterally: case report and review of the literature. J Trauma. 1993;34(3):458-460.

9. McLaughlin HD. Repair of ruptures through the larger tendons by removable staple. Arch Surg. 1946;52:547-556.

10. Rosenberg JM, Whitaker JH. Bilateral infrapatellar tendon rupture in a patient with jumper's knee. Am J Sports Med. 1991;19(1):94-95.
11. Siwek CW, Rao JP. Ruptures of the extensor mechanism of the knee joint. J Bone Joint Surg Am. 1981;63(6):932-937.

12. Van Glabbeek F, De Groof E, Boghemans J. Bilateral patellar tendon rupture: case report and literature review. J Trauma. 1992;33(5):790-792.

13. Webb LX, Toby EB. Bilateral rupture of the patella tendon in an otherwise healthy male patient following minor trauma. J Trauma. 1986;26(11):1045-1048.

14. Krackow KA, Thomas SC, Jones LC. A new stitch for ligament-tendon fixation. Brief note. J Bone Joint Surg Am. 1986;68(5):764-766.

15. Krackow KA, Thomas SC, Jones LC. Ligament-tendon fixation: analysis of a new stitch and comparison with standard techniques. Orthopedics. 1988;11(6):909-917.

16. Kelikian H, Riashi E, Gleason J. Restoration of quadriceps function in neglected tear of the patellar tendon. Surg Gynecol Obstet. 1957;104(2):200-204.

17. Mandelbaum BR, Bartolozzi A, Carney B. A systemic approach to reconstruction of neglected tears of the patellar tendon. Clin Orthop Relat Res. 1988 ;(235):268-271.

18. Levin PD. Reconstruction of the patellar tendon using a dacron graft: a case report. Clin Orthop Relat Res. 1976;(118):70-72.

19. Levy M, Goldstein J, Rosner M. A method of repair for quadriceps tendon or patellar ligament (tendon) ruptures without cast immobilization. Clin Orthop Relat Res. 1987;(218):297-301.

20. Hoisington SA, Taylor KW, Bachkosky DR. A technique for patella/ quadriceps tendon repair allowing immediate range of motion and full weight bearing. Am Acad of Orthop Surg. 1996.

21. Lindy PB, Boynton MD, Fadale PD. Repair of patellar tendon disruptions without hardware. J Orthop Trauma. 1995;9(3):238-243.

22. Marder RA, Timmerman LA. Primary repair of patellar tendon rupture without augmentation. Am J Sports Med. 1999;27(3):304-307.

23. Matava MJ. Patellar Tendon Ruptures. J Am Acad Orthop Surg. 1996;4(6):287-296.

24. Miskew DB, Pearson RL, Pankovich AM. Mersilene strip suture in repair of disruptions of the quadriceps and patellar tendons. J Trauma. 1980;20(10):867-872.

25. Persson K, Merkow RL, Templeman DC, et al. Patellar tendon rupture Description of a simplified operative method for a current therapeutic problem. Arch Orthop Trauma Surg. 1992;112(1):47-49.

26. Heegaard J, Leyvraz PF, Curnier A, et al. The biomechanics of the human patella during passive knee flexion. J Biomech. 1995;28(11):1265-1279.

27. Gelberman RH, Boyer MI, Brodt MD, et al. The effect of gap formation at the repair site on the strength and excursion of intrasynovial flexor tendons. An experimental study on the early stages of tendon-healing in dogs. J Bone Joint Surg Am. 1999;81(7):975-982. 